\title{
Inflammatory Properties and Adjuvant Potential of Synthetic Glycolipids Homologous to Mycolate Esters of the Cell Wall of Mycobacterium tuberculosis
}

\author{
Hermann Giresse Tima ${ }^{a}$ Juma'a Raheem Al Dulayymi ${ }^{d}$ Olivier Denis ${ }^{a}$ \\ Pauline Lehebel $^{\mathrm{a}} \quad$ Klarah Sherzad Baols $^{d}$ Mohsin Omar Mohammed ${ }^{d}$ \\ Laurent L'Homme ${ }^{b}$ Mohaned Mohammed Sahb ${ }^{d}$ Georges Potemberg ${ }^{a}$ \\ Sylvie Legrand ${ }^{b}$ Roland Lang ${ }^{\mathrm{e}}$ Rudi Beyaert $^{\mathrm{c}}$ Jacques Piette $^{\mathrm{b}}$ \\ Mark Stephen Baird ${ }^{d}$ Kris Huygen ${ }^{a}$ Marta Romano ${ }^{a}$ \\ ${ }^{a}$ Scientific Service Immunology, O.D. Communicable and Infectious Diseases, Scientific Institute of Public Health \\ (WIV-ISP), Brussels, 'b Laboratory of Virology and Immunology, GIGA-Signal Transduction, University of Liege, Liege, \\ and 'Inflammation Research Center VIB and Department for Biomedical Molecular Biology, Ghent University,

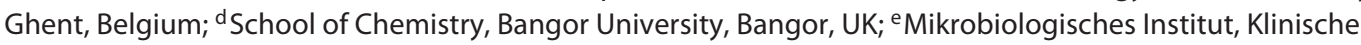 \\ Mikrobiologie, Immunologie und Hygiene, Universitätsklinikum Erlangen, Erlangen, Germany
}

\section{Keywords}

Mycobacterium tuberculosis · Glycolipids · Trehalose dimycolate $\cdot$ Trehalose monomycolate $\cdot$ Glucose monomycolate $\cdot$ Arabinose monomycolate Inflammasome . Adjuvant

\begin{abstract}
The cell wall of mycobacteria is characterised by glycolipids composed of different classes of mycolic acids (MAs; alpha-, keto-, and methoxy-) and sugars (trehalose, glucose, and arabinose). Studies using mutant $M t b$ strains have shown that the structure of MAs influences the inflammatory potential of these glycolipids. As mutant Mtb strains possess a complex mixture of glycolipids, we analysed the inflammatory potential of single classes of mycolate esters of the Mtb cell wall using 38 different synthetic analogues. Our results show that synthetic trehalose dimycolate (TDM) and treha-
\end{abstract}

\section{KARGER}

(c) 2016 S. Karger AG, Basel

E-Mail karger@karger.com

www.karger.com/jin lose, glucose, and arabinose monomycolates (TMM, GMM, and AraMM) activate bone marrow-derived dendritic cells in terms of the production of pro-inflammatory cytokines (IL-6 and TNF-a) and reactive oxygen species, upregulation of costimulatory molecules, and activation of NLRP3 inflammasome by a mechanism dependent on Mincle. These findings demonstrate that Mincle receptor can also recognise pentose esters and seem to contradict the hypothesis that production of GMM is an escape mechanism used by pathogenic mycobacteria to avoid recognition by the innate immune system. Finally, our experiments indicate that TMM and GMM, as well as TDM, can promote Th1 and Th17 responses in mice in an OVA immunisation model, and that further analysis of their potential as novel adjuvants for subunit vaccines is warranted.

(c) 2016 S. Karger AG, Basel 


\section{Introduction}

Mycobacterium tuberculosis (Mtb), the causative agent of tuberculosis, belongs to the single genus Mycobacterium of the Mycobacteriaceae family. Bacteria from this family are characterised by the presence in their cell wall of high molecular weight C-60 to C-90 2-alkyl-3-hydroxy fatty acids called mycolic acids (MAs). MAs are the major lipids constituting the mycobacterial cell wall $[1,2]$, present as free MAs [3] or esterified to different sugars. They are predominantly bound to the cell wall with arabinogalactan as penta-arabinose tetramycolates, but they are also present as non-bound solvent extractable esters, such as trehalose mono- and dimycolate (TMM and TDM), glucose monomycolate (GMM) and glycerol monomycolate $[1,2]$.

Among these components, trehalose 6,6'-dimycolate (TDM) - also known as cord factor - is the major and most studied mycolate ester. Indeed, in mouse models TDM can mimic certain aspects related to $M t b$ infection, including the production of pro-inflammatory cytokines $[4,5]$, the induction of granuloma and procoagulant activity [6-8]. In addition, TDM can delay phagolysosome fusion [9], is involved in tissue damage and necrosis, and possesses adjuvant properties [10]. However, the natural TDM used in these different studies was isolated from mycobacteria and consisted of a complex mixture of structurally related compounds composed of mycolates from different classes. Indeed, based on the functional group(s) present on their meromycolate chain, MAs are subdivided in different classes. The principal classes are the alpha-, keto-, and methoxyMAs. These classes differ considerably between mycobacterial species and strains [11], and appear to influence their virulence. For example, the attenuated vaccine strain $M$. bovis BCG-Pasteur lacks methoxy-MAs [12] and saprophytic mycobacteria such as M. smegmatis fail to produce oxygenated (keto- and methoxy-) MAs [2]. In contrast, highly pathogenic mycobacteria such as $M t b$ and $M$. leprae produce oxygenated MAs. Genetic inactivation of enzymatic pathways involved in the synthesis of oxygenated MAs resulted in in vivo-attenuated $M t b$ strains $[13,14]$. Therefore, the use of synthetic analogues can be useful to discriminate the contribution of different types of TDM or of mycolate esters in general to the pathogenicity of mycobacterial infection and to the inflammatory potential of these cell wall components. Indeed, the contribution of other mycolate esters found in the cell wall of $M t b$ such as TMM, GMM, and arabinose mycolates (AraMMs) in the activation of

Inflammatory Properties and Adjuvant Potential of Synthetic Mycolate Esters innate immune cells following mycobacterial infection is poorly defined.

TMM is known as a key precursor for the biosynthesis of TDM and has been reported to have some similarity with TDM in terms of inflammatory potential $[4,15,16]$. GMM has been demonstrated to be an antigen that is presented by CD1b molecules to T cells [17], but the activation of innate immune cells by GMM from $M t b$ is controversial. Indeed, it was reported that Mincle (macrophage inducible C-type lectin receptor) does not recognise GMM obtained by trehalase treatment of TDM [18], but that GMM of corynebacteria (C-32 mycolates in corynebacteria vs. C-60 to C-90 mycolates in mycobacteria) binds to Mincle and activates cells [19]. Mincle and MCL (macrophage C-type lectin) have been shown to be essential receptors for the recognition of $\operatorname{TDM}[18,20]$ and TMM [21], and hence crucial for the stimulation of cord factor-induced innate immune responses. On the other hand, the arabino-mycolate ester (a complex mixture of mono-AraMMs, tetra-arabinose tetra-mycolates, pentaarabinose tetra-mycolates, and hexa-arabinose tetra-mycolates) obtained by acid hydrolysis of the BCG cell wall skeleton was reported to induce the production of TNF- $\alpha$ by mechanisms dependent on the TLR-2/MyD88 pathway [22]. Finally, it has been shown that trehalose dibehenate (TDB), a short-acyl-chain structural analogue of TDM, activates the intracellular multiprotein complex called the NLRP3 inflammasome [23] and that this activation is essential for the Th17 responses induced by TDB [24]. For the moment, it is still unknown whether TMM, GMM, and arabinose mycolates can activate the inflammasome as well.

To address the relationship between the structure and the inflammatory power of mycolate esters and the signalling pathways involved, in this study we assayed 38 single synthetic mycolate ester isomers of high purity and known stereochemistry [25-28]. The mycolate esters tested here vary in terms of their carbohydrate moiety (trehalose, glucose, or arabinose) and in terms of the number, nature, and class of MAs bound to it. The selected mycolate esters are composed of MAs that are representative of the major classes found in the cell wall of $M t b$, namely alpha-, methoxy-, and keto-mycolates of either the cis or trans configuration and with different chain lengths. In addition, synthetic mycolate esters composed of alpha-mycolates containing an alkene group and homologous to those found in non-pathogenic mycobacteria (such as $M$. smegmatis) were also tested here for comparison purposes. Using murine bone marrow-derived dendritic cells (BMDCs), we examined in vitro the ability 
of these compounds to induce the production of pro-inflammatory cytokines (TNF- $\alpha$, IL-6, IL-12, and IL-1 $\beta$ ) and we identified the pathways involved in their inflammatory potential. We also confirmed their in vivo inflammatory potential and tested their adjuvant capacity to promote adaptive immune responses in an ovalbumin vaccination model.

\section{Material and Methods}

\section{Mice}

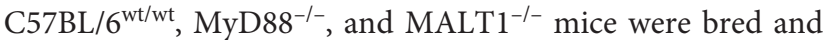
kept at the experimental animal facilities of WIV-ISP (Ukkel site, Brussels), complying with the Belgian legislation that transposes European Directive 2009/41/EC, repealing Directive 90/219/EC (EC, 2009). For some experiments, female C57BL/6 mice aged 6-8 weeks were purchased from Janvier Labs. Breeding pairs of $\mathrm{MyD} 8^{-/-}$mice were kindly provided by C. Desmet (Cellular and Molecular Physiology, GIGA-Research, ULg, Belgium). MALT1 ${ }^{-/-}$ mice were as described [29]. Bones (femurs and tibia) of $\mathrm{Mincle}^{-/-}$, $\mathrm{FcR} \gamma^{-1-}$, and $\mathrm{NLRP}^{-1-}$ mice were provided by R. Lang (University Clinics Erlangen, Germany) and B. Ryffel (CNRS, Orleans, France).

\section{Preparation of Glycolipid-Coated Plates}

Stocks of synthetic glycolipids (synthetised at Bangor University) [25-28, 30, 31], TDB (Invivogen), and natural TDM from $M t b$ (Adipogen) were solubilised in chloroform-methanol solution $(9: 1)$ at $5 \mathrm{mg} / \mathrm{mL}$. The different glycolipids were then dissolved in isopropanol (ISO) and coated on flat-bottomed culture plates (Greiner). After evaporation of the solvent, the plates were used directly or stored at $-20^{\circ} \mathrm{C}$. The final concentrations are indicated in the figure legends and Results section. For the experiments performed at equimolar concentrations, the molarity of natural TDM (a mix) was calculated using an estimated molecular weight of $2,754 \mathrm{~g} / \mathrm{mol}$. A possible negative effect of the tested glycolipids on cell viability was tested by Alamar blue assay and no increased mortality was observed under the test conditions.

\section{Generation of BMDCs}

BMDCs were generated as previously described [32]. Briefly, murine bone marrow from the femur and tibia was flushed with PBS and red blood cells were lysed with Sigma's lysing buffer. After lysis, cells were cultured $\left(5 \% \mathrm{CO}_{2}\right.$ at $\left.37^{\circ} \mathrm{C}\right)$ in a T75 flask in RPMI1640 medium (Life Technologies, Carlsbad, CA, USA) supplemented with $10 \mathrm{ng} / \mathrm{mL}$ recombinant murine GM-CSF (Immunotools), $5 \times 10^{-5} \mathrm{M} 2$-mercaptoethanol, $100 \mu \mathrm{g} / \mathrm{mL}$ gentamycin (GIBCO), $2 \mathrm{~mm}$ glutamine, $1 \mathrm{~mm}$ sodium pyruvate, non-essential amino acids (Thermo Fisher Scientific), and 10\% foetal calf serum (FCS; Greiner). Cells were differentiated for 6-7 days and the medium was replaced every 3 days. Ninety per cent of CD11c-positive cells were obtained after differentiation.

In vitro Stimulation with Glycolipids and Analysis of Samples

BMDCs were harvested after 7 days using a cell scraper, counted and seeded in glycolipid-coated plates at a concentration of $10^{6}$ cells/mL for different time periods. Culture supernatants were collected after $24 \mathrm{~h}$ and the content of pro-inflammatory cytokines
TNF- $\alpha$, IL-12p40, IL- $1 \beta$, and IL-6 was determined by ELISA (eBiosciences).

The expression of costimulatory molecules was analysed after $48 \mathrm{~h}$ of incubation with the different compounds. Cells were labelled with anti-mouse CD80, anti-mouse CD86, and anti-mouse major histocompatibility complex class II (MHC-II) monoclonal antibodies (eBiosciences). Fluorescence was analysed using a FACSCalibur flow cytometer and CELL-QUEST software (BD Biosciences).

\section{HEK-Blue mMincle Stimulation}

HEK (human embryonic kidney)-blue mMincle cells (Invivogen) stably transfected with murine C-type lectin receptor mincle gene, as well as the genes of the Mincle-NF- $\kappa$ B signalling pathway and secreted embryonic alkaline phosphatase (SEAP) under the control of NF- $\kappa \mathrm{B}$ activation, were used to analyse the activation of the Mincle receptor by the compounds. HEK-blue mMincle cells were cultured in Dulbecco's modified Eagle's medium containing $4.5 \mathrm{~g} / \mathrm{L}$ glucose and supplemented with $10 \%$ FCS, selective antibiotics, and $2 \mathrm{~mm}$ glutamine. Cells were passed in a T75 flask when $70-80 \%$ confluence was reached. For the stimulation, cells were seeded in 96-well plates at a density of $5 \times 10^{4}$ cells/well for $24 \mathrm{~h}$. Quanti-blue substrate (Invivogen) was used for the detection of SEAP in the supernatant according to the manufacturer's instructions.

\section{Inflammasome Assays}

BMDCs at $1 \times 10^{6}$ cells $/ \mathrm{mL}$ were primed for $3 \mathrm{~h}$ with $1 \mu \mathrm{g} / \mathrm{mL}$ of Escherichia coli K12 ultra-pure LPS (Invivogen) prior to stimulation with glycolipids (on coated plates) and controls. Cells were stimulated in triplicate with $5 \mathrm{mM}$ ATP (for $1 \mathrm{~h}$ ) or $10 \mu \mathrm{g} / \mathrm{mL}$ of MA esters (for $5 \mathrm{~h}$ ). For the inhibition studies, glibenclamide (50 $\mu \mathrm{M}$; Sigma), ebselen (10 $\mu \mathrm{M}$; Sigma), cytochalasin D ( $1 \mu \mathrm{M}$; Sigma), CA-074 methyl ester (10 $\mu \mathrm{M}$; Sigma), and Z-VAD-FMK (10 $\mu \mathrm{M}$; Invivogen) were added $1 \mathrm{~h}$ prior to glycolipid stimulation. The effect of these inhibitors on cell viability was tested by Alamar blue or MTT assay and no increased mortality was observed under the test conditions. IL- $1 \beta$ cytokine production in cell-free supernatant was evaluated by ELISA (eBiosciences).

For the detection of activated caspase- 1 in supernatants, cells were stimulated in FCS-free medium. Triplicate samples were pooled and the proteins were concentrated by methanol-chloroform precipitation. Briefly, $500 \mu \mathrm{L}$ of supernatant was mixed with $500 \mu \mathrm{L}$ of methanol and $125 \mu \mathrm{L}$ of chloroform, vortexed and centrifuged. The aqueous phase was collected, washed with methanol, mixed and centrifuged. The supernatant was discarded and the pellet was dried, reconstituted in SDS sample buffer (75 mM Tris$\mathrm{HCl}, \mathrm{pH}$ 6.8, $100 \mathrm{mM}$ DTT, 2\% SDS, 0.01\% bromophenol blue and $10 \%$ glycerol) and heated for $5 \mathrm{~min}$ at $95^{\circ} \mathrm{C}$. Proteins were separated by electrophoresis for $1.5 \mathrm{~h}$ at $100 \mathrm{~V}$ in $15 \%$ Tris-Glycine Gel and transferred to a nitrocellulose membrane using a semi-dry system (Amersham Bioscience). Three per cent BSA and TBS-Tween 20 were used as blocking and washing buffer, respectively. Monoclonal mouse anti-caspase-1 p20 Casper-1 (Adipogen) was used as the primary antibody and polyclonal goat anti-mouse IgG HRP (R\&D Systems) as a secondary antibody. The revelation was done by applying ECL substrate (GE Healthcare Life Sciences) on the membrane and pictures were taken using an ImageQuant LAS 4,000 device. 
Reactive Oxygen Species Assay

Reactive oxygen species (ROS) were analysed with cell-permeant $2^{\prime}, 7^{\prime}$-dichlorodihydrofluorescein diacetate $\left(\mathrm{H}_{2} \mathrm{DCFDA}\right)$ assay according to the manufacturer's protocol (Thermo Fisher Scientific). Briefly, BMDCs $\left(10^{6}\right.$ cells $\left./ \mathrm{mL}\right)$ were pre-incubated for 30 min in PBS to allow the dye to enter the cells, washed and seeded in 24-well coated plates for $4 \mathrm{~h}$. ROS levels were analysed by flow cytometry. The results were analysed using a FACSCalibur flow cytometer and CELL-QUEST software (BD Biosciences).

\section{Footpad Immunisation with Ovalbumin}

Water in oil in water $(\mathrm{w} / \mathrm{o} / \mathrm{w})$ emulsions were prepared by minor modification of a previously described protocol [33]. Briefly, glycolipids were dissolved in $30 \%$ incomplete Freund's adjuvant (IFA) and vortexed vigorously. Next, $0.1 \mathrm{M}$ PBS was added and vortexed again vigorously to make a water in oil emulsion, and finally PBS containing $0.2 \%$ of Tween 80 and the required amount of ovalbumin was added to the water in oil preparation to make a w/o/w emulsion. Groups of 4-5 mice were injected subcutaneously in both hind footpads with $25 \mu \mathrm{L}$ of the emulsion containing $30 \%$ IFA, $5 \mu \mathrm{g}$ of glycolipids, and $25 \mu \mathrm{g}$ of ovalbumin (Invivogen). Footpad swelling in individual mice was measured with a caliper before and at several time points after injection.

\section{Analysis of Local Footpad Inflammation}

One week after the administration of the w/o/w samples, the mice were sacrificed and the tissues of the hind footpads were collected and digested using collagenase and DNAse (Sigma). Cells were collected and strained with a $100-\mu$ m nylon cell strainer (BD Biosciences). Total RNA was isolated from footpad cells with Trireagent (Sigma-Aldrich) according to the manufacturer's instructions. cDNA was prepared using the GoScript Reverse Transcriptase system (Promega). qRT-PCR was performed on Stratagene 3000p using the GoTaq qPCR Master mix (Promega) according to the manufacturer's instructions. The forward and reverse primers used are described below. GAPDH was used as the reference housekeeping gene for the normalisation. Additionally, footpad cells were incubated with Ly6G-Ly6C PE and viability dye V450 (eBiosciences), and the fluorescence was analysed with a FACSverse device.

\section{Primers}

CXCL1 forward: 5'-CCGAAGTCATAGCCACACTCAA-3'; CXCL1 reverse: $5^{\prime}$-GCAGTCTGTCTTCTTTCTCCGTTAC-3'; IL- $1 \beta$ forward: $5^{\prime}$-TTGACGGACCCCAAAAGAT-3'; IL- $1 \beta$ reverse: $5^{\prime}$-AGCTGGATGCTCTCATCAGG-3'; IL-12p35 forward: 5'-CCTCGGCATCCAGC-3'; IL-12p35 reverse: 5' -TCTGGCCGTCCTCA-3'; TNF- $\alpha$ forward: $5^{\prime}$-CATCTTCTCAAAATTCGAGTGACAA-3'; TNF- $\alpha$ reverse: $5^{\prime}$-TGGGAGTAGACAAGGTACAACCC-3'; GAPDH forward: $5^{\prime}$-TCGGCCTTGACTGTGCCGTT-3'; GAPDH reverse: $5^{\prime}$-TCCCAGCCTCGTCCCGTAG$\mathrm{AC}-3^{\prime}$.

\section{Analysis of Adaptive Immune Responses}

One week after the subcutaneous administration of the different w/o/w preparations, mice were sacrificed, popliteal and inguinal lymph nodes were removed and passed through a $100-\mu \mathrm{m}$ nylon cell strainer (BD Biosciences). Cells were counted and stimulated in RPMI medium supplemented with $5 \times 10^{-5} \mathrm{M} 2$-mercaptoethanol, antibiotics, and 10\% FCS in round-bottomed 96-well

Inflammatory Properties and Adjuvant

Potential of Synthetic Mycolate Esters plates with $5 \mu \mathrm{g} / \mathrm{mL}$ of ovalbumin, culture medium as a negative control and Concanavalin A ( $4 \mu \mathrm{g} / \mathrm{mL}$; Sigma) as a positive control. Cell-free culture supernatants were harvested after 24 and $72 \mathrm{~h}$ of incubation at $37^{\circ} \mathrm{C}, 5 \% \mathrm{CO}_{2}$. Levels of IL-2 were measured in 24-h supernatants by ELISA (eBiosciences). Levels of IFN- $\gamma$ and IL-17A were determined in 72-h supernatants by ELISA (BD Pharmingen and eBiosciences, respectively).

\section{Statistical Analyses}

Statistical analyses were performed using GraphPad Prism 6 software (GraphPad Software, La Jolla, CA, USA). For the statistical analysis of the in vitro experiments, data from 3-4 independent experiments were pooled and tested for a Gaussian distribution with the D'Agostino Pearson test followed by pairwise comparisons performed by the Mann-Whitney test. For the analysis of the in vivo experiments, pairwise comparisons were performed by Mann-Whitney test. For all analyses, $p<0.05$ was considered statistically significant.

\section{Results}

\section{Synthetic TDMs and TMMs Are Inflammatory}

To study the influence of the class of mycolates and the number of mycolate chains on the inflammatory potential of trehalose mycolate esters, 17 TDMs and 13 TMMs were synthetised (Table 1, TDM and TMM columns) $[25-28,30,31]$. These compounds vary in terms of their MA class (alpha-, methoxy-, keto-), the configuration of their cyclopropanation (cis- vs. trans-), and the number of MAs bound to trehalose (dimycolates vs. monomycolates). BMDCs were incubated for $24 \mathrm{~h}$ with the different synthetic TMMs, TDMs, evaporated ISO as a negative control, trehalose-6,6-dibehenate (TDB), or TDM isolated from $M t b$ (TDM natural mix) as positive controls. The production of the pro-inflammatory cytokines IL- 6 and TNF- $\alpha$ was evaluated by ELISA in culture supernatants and the results obtained for 10 TDMs and 9 TMMs at equimolar concentrations are shown in Figure 1, while results obtained at $10 \mu \mathrm{g} / \mathrm{mL}$ for 16 TDMs and $11 \mathrm{TMMs}$ are shown in online supplementary Table S1 (see www. karger.com/doi/10.1159/000450955 for all online suppl. material). All the tested synthetic TMMs and TDMs stimulated the production of TNF- $\alpha$ and IL-6. Interestingly, when dose-response analysis was performed with pairs of TMMs and TDMs composed of the same mycolate moiety, we observed that the number of mycolate chains bound to trehalose influenced the level of TNF- $\alpha$ and IL- 6 produced. Indeed, as shown in Figure 1a and b, at equimolar concentrations the cis-methoxy TMM KB51 (monomycolate) induced TNF- $\alpha$ and IL- 6 levels that were 2- to 3 -fold lower than those achieved by stimulation with the corresponding cis-methoxy TDM KB52 (dimycolate). 
Table 1. Structure, class, code names, and reference to synthesis of the tested synthetic mycolate ester

\begin{tabular}{llll}
\hline $\begin{array}{l}\text { Class of } \\
\text { mycolic acid }\end{array}$ & Structure of mycolic acid & TDM & TMM \\
\hline
\end{tabular}

Alpha mycolic acids

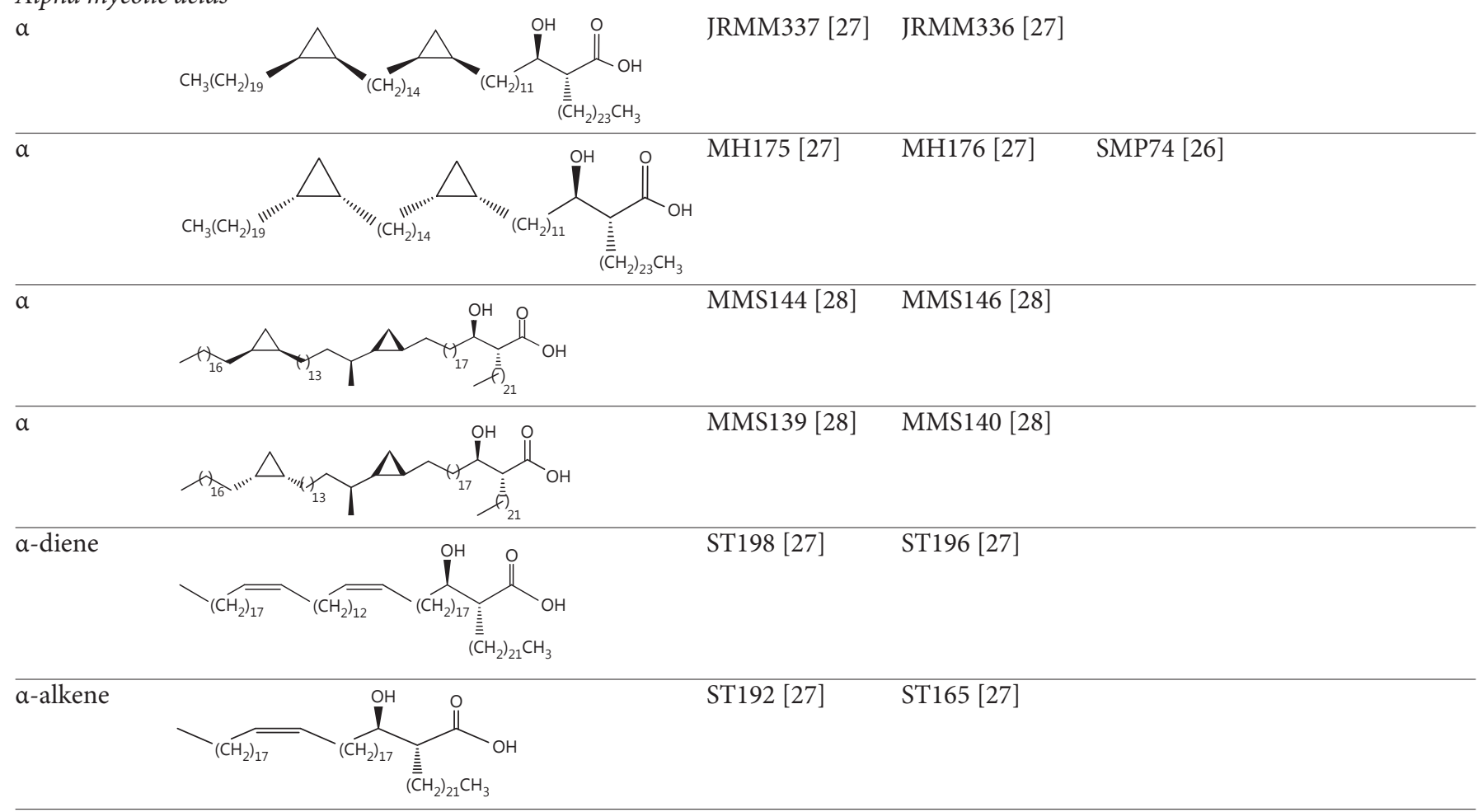

Keto mycolic acids

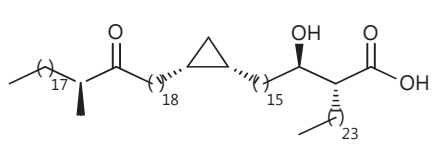

$\mathrm{AD} 132[27] \quad \mathrm{AD} 134[27]$

cis-keto

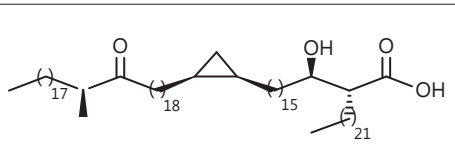

cis-keto

RT137 [31]

trans-keto

RT136 [27]

cis-keto 
Table 1 (continued)

\begin{tabular}{|c|c|c|c|c|c|}
\hline $\begin{array}{l}\text { Class of } \\
\text { mycolic acid }\end{array}$ & Structure of mycolic acid & TDM & TMM & GMM & AraMM \\
\hline trans-keto & & & & SMP75 [26] & MOD23 [25] \\
\hline $\begin{array}{l}\text { Methoxy mycol } \\
\text { cis-methoxy }\end{array}$ & lic a & AD104 [27] & AD118 [27] & & \\
\hline cis-methoxy & & KB52 [31] & KB51 [31] & SMP73 [26] & MOD16 [25] \\
\hline cis-methoxy & & KB91 [27] & & SMP71 [26] & \\
\hline cis-methoxy & & JRMM324 [27] & JRMM319 [27] & & MOD18 [25] \\
\hline trans-methoxy & & RT126 [27] & & & \\
\hline
\end{tabular}

Hydroxy mycolic acids

Hydroxy

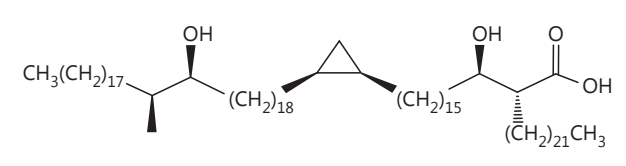

RT141 [27]

Mixed mycolic acids

Mixed

methoxy and

$\alpha$-mycolates

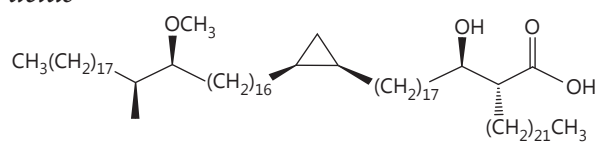

KB55 [31]<smiles>CCCC1CC1CCC(C)C1CC1CCNC(O)C(=O)O</smiles>

TNF- $\alpha$ levels induced by KB51 were comparable to those induced by TDB, while IL- 6 levels induced by KB51 were lower than those obtained by TDB. This influence of the number of mycolate chains on the intensity of the inflammatory response was observed for a total of 9 TMM/TDM pairs representative of the different classes and configurations of mycolates present in mycobacteria (Fig. 1c). Indeed, statistically significant differences in the levels of TNF- $\alpha$ (Fig. 1c) and IL-6 (data not shown) were observed when comparing the data obtained for a given TDM with 
the data obtained for the corresponding TMM (same mycolate moiety). In addition, the majority of the tested TDM compounds induced significantly more TNF- $\alpha$ compared to TDB (statistical analysis not shown). Only the dialkene TDM (ST198) and the cis-keto TDM (AD132) induced TNF- $\alpha$ levels comparable to those induced by TDB (Fig. 1c). Concerning the influence of the class of MAs on the levels of inflammatory responses induced by

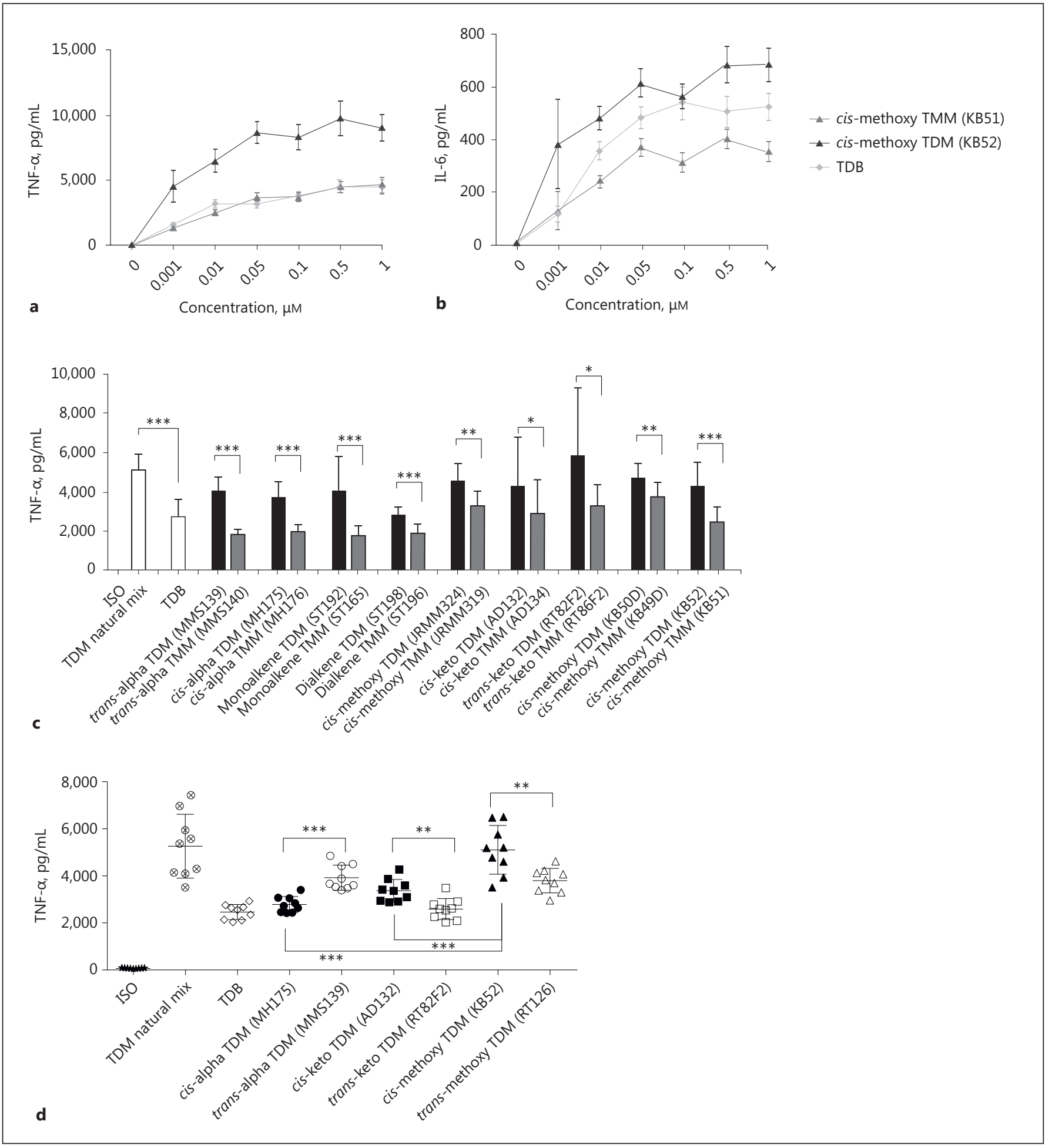

(For legend see next page.) 
TDM, 8 synthetic TDMs representative of the different classes and configurations of mycolates present in mycobacteria were selected and a dose-response analysis was performed. All the tested synthetic compounds induced significant TNF- $\alpha$ production already at the lowest 0.001 $\mu \mathrm{M}$ concentration tested and a response plateau was observed at $0.1-1 \mu \mathrm{M}$ (online suppl. Fig. S1). To compare the responses induced with TDMs from the same class but of different configurations (cis- vs. trans-), data obtained in 3 independent experiments at $1 \mu \mathrm{M}$ with alpha, keto, or methoxy TDMs of cis- or trans-configuration were pooled and the differences were analysed statistically (Fig. 1d). Results indicated that the tested trans-alpha TDM (MMS139) was more inflammatory than the tested cisalpha TDM (MH175), while for the oxygenated mycolates cis- compounds were more inflammatory than trans- compounds (Fig. 1d). When the results obtained for cis- compounds are statistically compared, cis-methoxy-compound (KB52) induces higher TNF- $\alpha$ levels compared to cis-alpha TDM (MH175) or cis-keto TDM (AD132).

\section{Synthetic AraMMs and GMMs Are Inflammatory}

We next assessed the importance of the sugar moiety on the inflammatory potential of synthetic glycolipids. For that purpose, levels of pro-inflammatory cytokines produced by a cis-methoxy mycolate bound to trehalose (TDM-KB52 or TMM-KB51), or to glucose (GMMSMP73) or arabinose (AraMM-MOD16) were evaluated. As shown in Figure 2a-d, significant TNF- $\alpha$, IL-6, IL$12 \mathrm{p} 40$, and IL-1 $\beta$ levels were measured in BMDC culture supernatants with all the tested compounds after $24 \mathrm{~h}$ of incubation. At the highest concentration tested $(1 \mu \mathrm{M})$, TDB induced significantly more IL- 6 , IL- $1 \beta$, TNF- $\alpha$, and IL-12p40 than cis-methoxy AraMM-MOD16 and TMM-

Fig. 1. Synthetic TMM and TDM esters stimulate the in vitro production of TNF- $\alpha$ and IL- 6 by BMDCs. $\mathbf{a}, \mathbf{b}$ BMDCs derived from C57BL/6 mice were stimulated for $24 \mathrm{~h}$ in triplicate cultures with increasing concentrations of plate-coated synthetic cis-methoxy TMM-KB51, cis-methoxy TDM-KB52, or TDB. The supernatants were harvested from separate wells and the volume of pro-inflammatory cytokines (TNF- $\alpha$ and IL-6) was determined by sandwich ELISA. Results are expressed as the mean amount of cytokines \pm SD of 3 independent experiments. c Separate BMDC cultures derived from C57BL/6 mice were stimulated for $24 \mathrm{~h}$ in triplicate with 9 pairs of synthetic TDM versus corresponding TMM esters at $0.1 \mu \mathrm{M}$, with evaporated ISO as a negative control and TDB or natural TDM at the same concentration as positive controls. The supernatants were harvested and the amount of TNF- $\alpha$ was determined by sandwich ELISA. Results are expressed as the mean
KB51, whereas GMM-SMP73 - compared to TDB - induced comparable levels of IL- $1 \beta$ and TNF- $\alpha$ but less IL12 p40 and IL-6. Finally, TDM-KB52 induced similar levels of IL-6, IL-1 $\beta$, and IL-12p40 but higher TNF- $\alpha$ levels as compared to levels achieved by TDB. Overall, these data indicate that AraMM-MOD16 is the weakest inducer of pro-inflammatory cytokines in comparison to TDMKB52, TMM-KB51, and GMM-SMP73. In an additional experiment, we also compared the inflammatory potential of 5 synthetic GMMs and 3 AraMMs composed of mycolates from different classes and configuration (see online suppl. Fig. S2). Notably, all the tested GMMs induced TNF- $\alpha$ levels at least comparable to those obtained with TDB (see online suppl. Fig. S2). In contrast, the TNF- $\alpha$ levels induced with the 3 different synthetic AraMMs were lower than those induced with TDB or the tested GMMs. Thus, AraMMs are the weakest inducers of pro-inflammatory cytokines and TDMs the strongest inducers, while TMMs and GMMs induce responses comparable in magnitude to those observed after stimulation with TDB.

As a second read-out for the activation of BMDCs, we measured the surface expression of the costimulatory molecules CD86 and CD80, MHC-II molecules, and production of ROS induced by incubation with the synthetic glycolipids. Expression of CD86, CD80, and MHC-II was increased after $48 \mathrm{~h}$ of incubation with TDM-KB52, TMM-KB51, GMM-SMP73, AraMM-MOD16, and LPS and TDB controls as compared to non-stimulated BMDCs (Fig. 2e). AraMM-MOD16 (and LPS) stimulated CD86 expression to a lesser extent than the other mycolate esters. Expression of CD80 and MHC-II was increased by all 4 compounds to the same level as by TDB. TDM-KB52, TMM-KB51, and GMM-SMP73 all significantly induced the production of intracellular ROS, while

amount of TNF- $\alpha \pm$ SD and data of 4 representative experiments have been pooled. Statistical analysis of results obtained for the TDM/TMM pairs was performed by the Mann-Whitney test. ${ }^{*} p<0.05,{ }^{* *} p<0.01$, and ${ }^{* * *} p<0.001$. d Separate BMDC cultures derived from C57BL/6 mice were stimulated for $24 \mathrm{~h}$ in triplicate with single TDMs representative of the different classes and configurations of mycolates present in mycobacteria at $1 \mu \mathrm{M}$, with evaporated ISO as a negative control and TDB or natural TDM at the same concentration as positive controls. The supernatants were harvested and the amount of TNF- $\alpha$ was determined by sandwich ELISA. Results are expressed as the mean amount of TNF- $\alpha$ $\pm \mathrm{SD}$ and data of 3 representative experiments have been pooled. Statistical analysis of the results of the different groups was performed by the Mann-Whitney test. ${ }^{* *} p<0.01$ and ${ }^{* * *} p<0.001$.
Inflammatory Properties and Adjuvant Potential of Synthetic Mycolate Esters
J Innate Immun 2017;9:162-180 DOI: $10.1159 / 000450955$ 


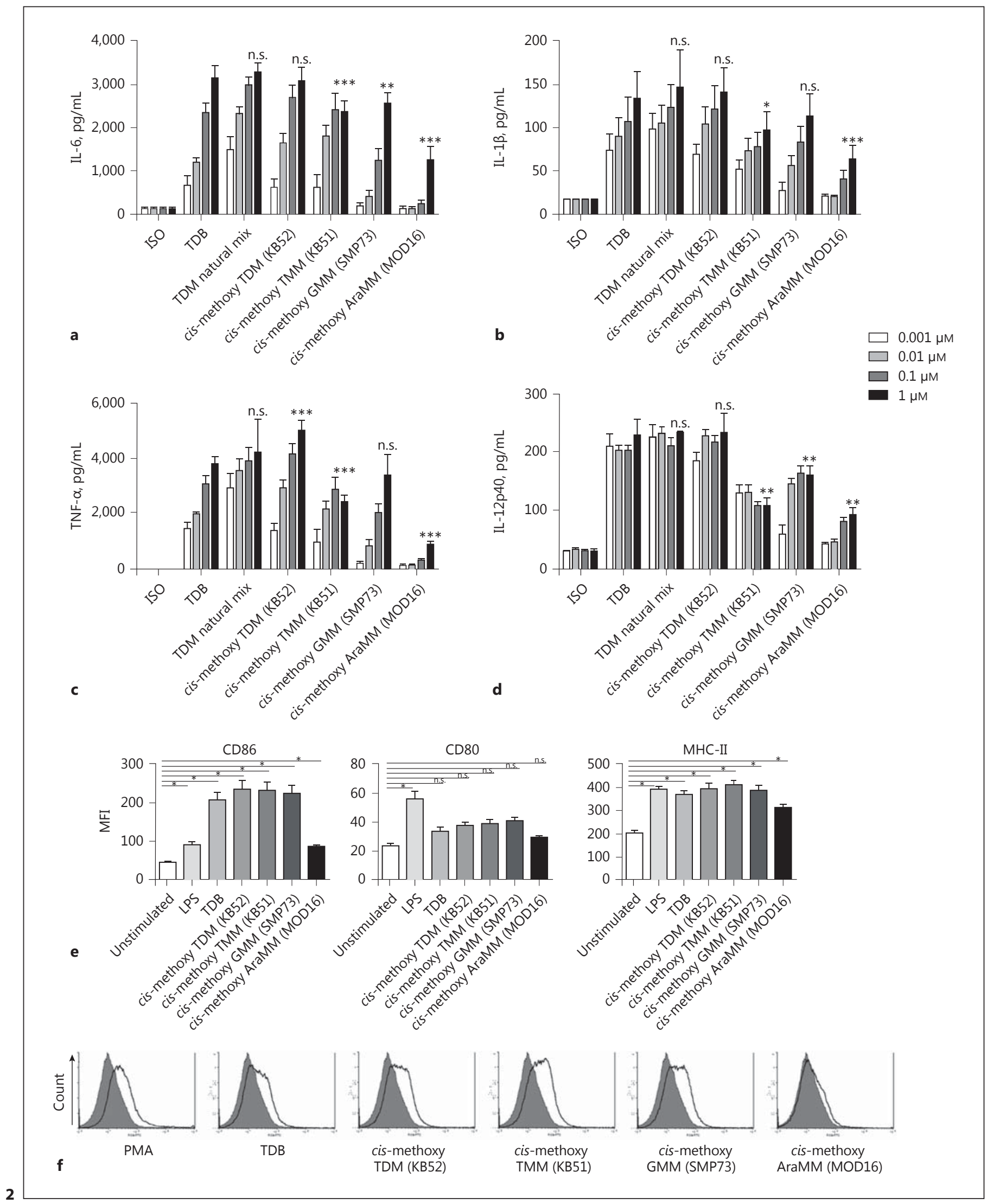

(For legend see next page.) 
the weakest response was again observed for the synthetic AraMM tested (Fig. 2f).

\section{Synthetic AraMMs and GMMs Activate BMDCs by Mechanisms Dependent on the C-Type Lectin Receptor Mincle}

Several receptors have been reported to interact with mycolate esters. For example, recognition of natural arabinose mycolate esters has been described to be dependent on MyD88 and TLR-2 [22], while the C-type lectin receptor Mincle and MCL receptor have been associated with recognition of cord factor, its structural analogue TDB, TMM, and GMM from corynebacteria [18-21, 34]. In order to identify the signalling pathways involved in the inflammatory responses observed with the synthetic compounds, BMDCs were generated from C57BL/6 $\mathrm{WT}, \mathrm{Myd} 88^{-/-}, \mathrm{Mincle}^{-/-}, \mathrm{FcR} \gamma^{-/-}$, and $\mathrm{MALT1}^{-/-}$mice. MALT1 and FcR $\gamma$ are 2 proteins involved in the MincleNF- $\kappa B$ activation pathway. As shown in Figure $3 a$, TNF- $\alpha$ production induced by LPS was dependent on Myd88 but was independent of Myd88 for the synthetic TDM-KB52, TMM-KB51, GMM-SMP73, TDB, and natural TDM mix, and also for AraMM-MOD16, in contrast to what was reported for natural AraMM isolated from BCG [22]. TNF- $\alpha$ production induced by LPS was, as expected, independent of Mincle, FcR $\gamma$, and MALT1, while complete inhibition of TNF- $\alpha$ production was observed in BMDCs from $\mathrm{Mincle}^{-/-}, \mathrm{FcR} \gamma^{-/-}$, and MALT1 ${ }^{-/-}$mice after stimulation with synthetic TDM-KB52, TMM-KB51, GMMSMP73, TDB, natural TDM mix and with AraMMMOD16 (Fig. 3b, c). Similar results were obtained when piceatannol was used as an inhibitor of spleen tyrosine kinase, a protein involved in NF- $\kappa \mathrm{B}$ activation by Mincle

Fig. 2. Synthetic GMMs and AraMMs activate BMDCs. a-d BMDC cultures from C57BL/6 mice were stimulated for $24 \mathrm{~h}$ in triplicate with escalating concentrations of plate-coated synthetic mycolate esters composed of the same cis-methoxy mycolate bound twice to trehalose (TDM-KB52) or once to trehalose (TMM-KB51), glucose (GMM-SMP73), arabinose (AraMMMOD16), or controls (natural TDM mix, TDB, or evaporated ISO). The supernatants were harvested and the volumes of proinflammatory cytokines (IL-6, a; IL-1 $\beta$, b; TNF- $\alpha$, c; IL-12p40, d) were determined by ELISA. Results are expressed as the mean amount of cytokines $\pm \mathrm{SD}$ ( $n=4$ independent experiments). Statistical analysis on the results obtained for synthetic compounds and natural TDM mix compared to TDB was performed by the Mann-Whitney test. n.s., not significant. ${ }^{* *} p<0.01$, and ${ }^{* * *} p<$ 0.001. e Upregulation of costimulatory molecules (CD86 and CD80) and MHC-II was evaluated by flow cytometric analysis after $48 \mathrm{~h}$ of stimulation with $10 \mu \mathrm{g} / \mathrm{mL}$ of synthetic mycolate esters (data not shown). In addition, using the HEK-blue murine Mincle reporter cell line (Invivogen), we confirmed that synthetic glucose and arabinose monomycolates are Mincle agonists (Fig. 3d). Comparable levels of NF- $\kappa B-$ inducible SEAP were induced by TDM-KB52, TMMKB51, GMM-SMP73, and TDB at concentrations as low as $0.001 \mu \mathrm{M}$, while 100-fold-higher concentrations of AraMM-MOD16 were needed to stimulate comparable SEAP levels, suggesting a weaker interaction with Mincle. Similar results were obtained with all the tested synthetic TDMs, TMMs, GMMs, and AraMMs (see online suppl. Fig. S3 for the TDM results).

In summary, these results indicate that synthetic GMMs and AraMMs activate BMDCs by mechanisms that are independent of the Myd88 pathway but dependent on the Mincle-FcR $\gamma$-Syk MALT1 pathway, demonstrating that the C-type lectin Mincle receptor can also recognise MAs from mycobacteria bound to glucose and arabinose.

\section{GMMs and AraMMs Activate the NLRP3}

\section{Inflammasome}

It was previously shown that the production of IL- $1 \beta$ induced by natural TDM (mix) and TDB is associated with activation of the NLRP3 inflammasome [23]. The inflammasome is a multiprotein platform which mediates the maturation of caspase-1. Mature caspase- 1 cleaves pro-IL-1 $\beta$ and pro-IL-18, and induces the secretion of mature IL-1 $\beta$ and IL-18 [35]. The activation of the inflammasome by TDB is dependent on Mincle and has been shown to be essential for its ability to induce IL-17 responses [24]. As we found that synthetic GMMs and AraMMs also bind to Mincle and induce the production

composed of the same cis-methoxy mycolate bound twice to trehalose (TDM-KB52) or once to trehalose (TMM-KB51), glucose (GMM-SMP73), arabinose (AraMM-MOD16), or controls (unstimulated, LPS $100 \mathrm{ng} / \mathrm{mL}$, or TDB $10 \mu \mathrm{g} / \mathrm{mL}$ ). Results are the mean \pm SD and representative of at least 3 independent experiments. Statistical analysis on results obtained for synthetic compounds, TDB, or LPS compared to unstimulated cells was performed by the Mann-Whitney test: n.s., not significant. ${ }^{*} p<0.05$. f BMDCs were treated for 30 min with $2^{\prime}, 7^{\prime}$-dichlorodihydrofluorescein diacetate (H2DCFDA) and stimulated for $4 \mathrm{~h}$ with $10 \mu \mathrm{g} /$ $\mathrm{mL}$ of synthetic mycolate esters composed of the same cis-methoxy mycolate bound twice to trehalose (TDM-KB52) or once to trehalose (TMM-KB51), glucose (GMM-SMP73), arabinose (AraMMMOD16), or controls (unstimulated, PMA $100 \mathrm{ng} / \mathrm{mL}$, or TDB $10 \mu \mathrm{g} / \mathrm{mL}$ ). The production of ROS by the tested compound (black line) was measured by flow cytometry and compared to unstimulated cells (grey shading).
Inflammatory Properties and Adjuvant Potential of Synthetic Mycolate Esters
J Innate Immun 2017;9:162-180 DOI: $10.1159 / 000450955$ 


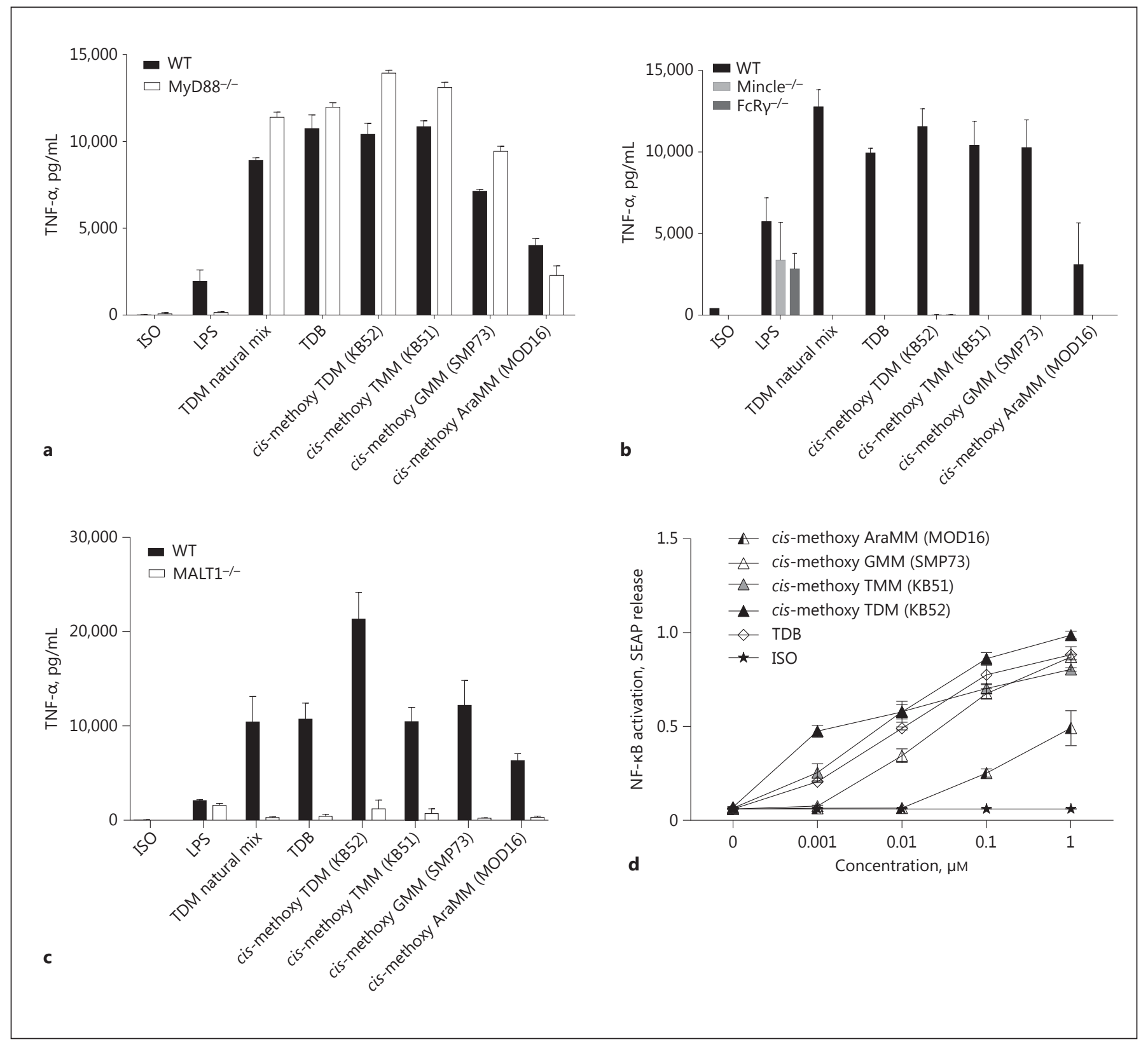

Fig. 3. Synthetic GMMs and AraMMs activate BMDCs by Mincle-dependent mechanisms. a-c BMDCs from C57BL/6 WT,

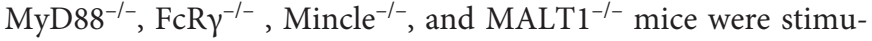
lated in triplicate with $10 \mu \mathrm{g} / \mathrm{mL}$ of different synthetic mycolate esters, TDB, natural TDM mix, or negative control (evaporated ISO) for $24 \mathrm{~h}$. The supernatants were analysed for their content in pro-inflammatory cytokines (TNF- $\alpha$ ) by ELISA. Results are ex- pressed as the mean volume of TNF- $\alpha \pm$ SD and are representative of at least 4 independent experiments. d HEK293 reporter cells expressing murine Mincle were used to evaluate binding to the Mincle receptor. SEAP release after NF- $\kappa$ B activation was evaluated by colorimetric assay using Quantie-blue substrate and by measuring the OD at $655 \mathrm{~nm}$. of ROS species, we next analysed whether these compounds could also activate the inflammasome. A selected series of 4 mycolate esters composed of the same cis-methoxy mycolate were tested, i.e. TDM-KB52, TMM-KB51,
GMM-SMP73, and AraMM-MOD16. BMDCs were primed with LPS $3 \mathrm{~h}$ prior to incubation for $5 \mathrm{~h}$ with the tested compounds and controls. As shown in Figure 4a, synthetic cis-methoxy TDM-KB52, TMM-KB51, GMM- 


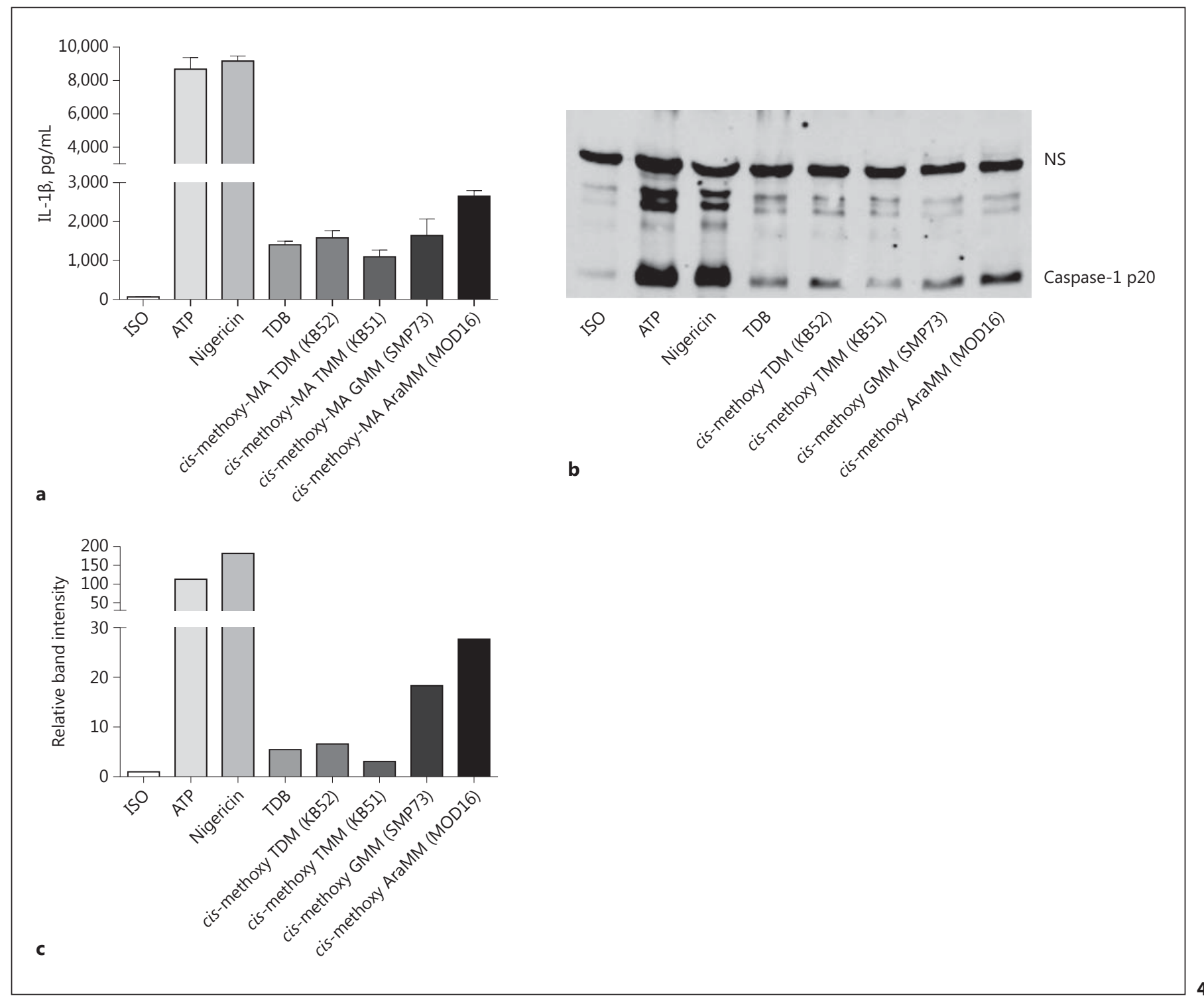

(For rest of figure 4 and legend see next page.)

SMP73, and AraMM-MOD16 all stimulated IL- $1 \beta$ secretion by LPS-primed BMDCs to levels comparable to those achieved by natural TDM and TDB. Because the secretion of IL- $1 \beta$ is dependent on the maturation of caspase- 1 , we also analysed the secretion of the active caspase- 1 p20 subunit in the supernatant and found that stimulation of LPS-primed BMDCs by TDM-KB52, TMM-KB51, GMM-SMP73, or AraMM-MOD16 was sufficient to induce caspase- 1 maturation (Fig. 4b, c). Blocking of caspase activity with the pan-caspase-inhibitor Z-VADFMK strongly reduced the secretion of IL-1 $\beta$ (Fig. $4 \mathrm{~d}$ ), while, as expected, levels of secreted TNF- $\alpha$ were only marginally reduced following Z-VAD-FMK addition
(Fig. 4e). Overall, these results indicate that synthetic TMM, GMM, and AraMM can also induce a caspase-1/ inflammasome-dependent secretion of IL- $1 \beta$ in BMDCs. Using BMDCs derived from $\mathrm{NLRP}^{-/-} \mathrm{KO}$ and caspase- $1 / 11^{-/-}$mice, we observed that IL- $1 \beta$ induction by the tested compounds was completely abrogated in the absence of NLRP3 and significantly reduced in the absence of caspase- $1 / 11^{-/-}$(Fig. $4 \mathrm{f}$ ), demonstrating that synthetic TMM, GMM, and AraMM induce the production of IL- $1 \beta$ by NLRP3-dependent mechanisms (Fig. 4f). The fact that only levels of IL- $1 \beta$ but not of TNF- $\alpha$ were affected by the genetic deletion of NLRP 3 or caspase-1/11 confirms that these deletions only impact inflammasome 


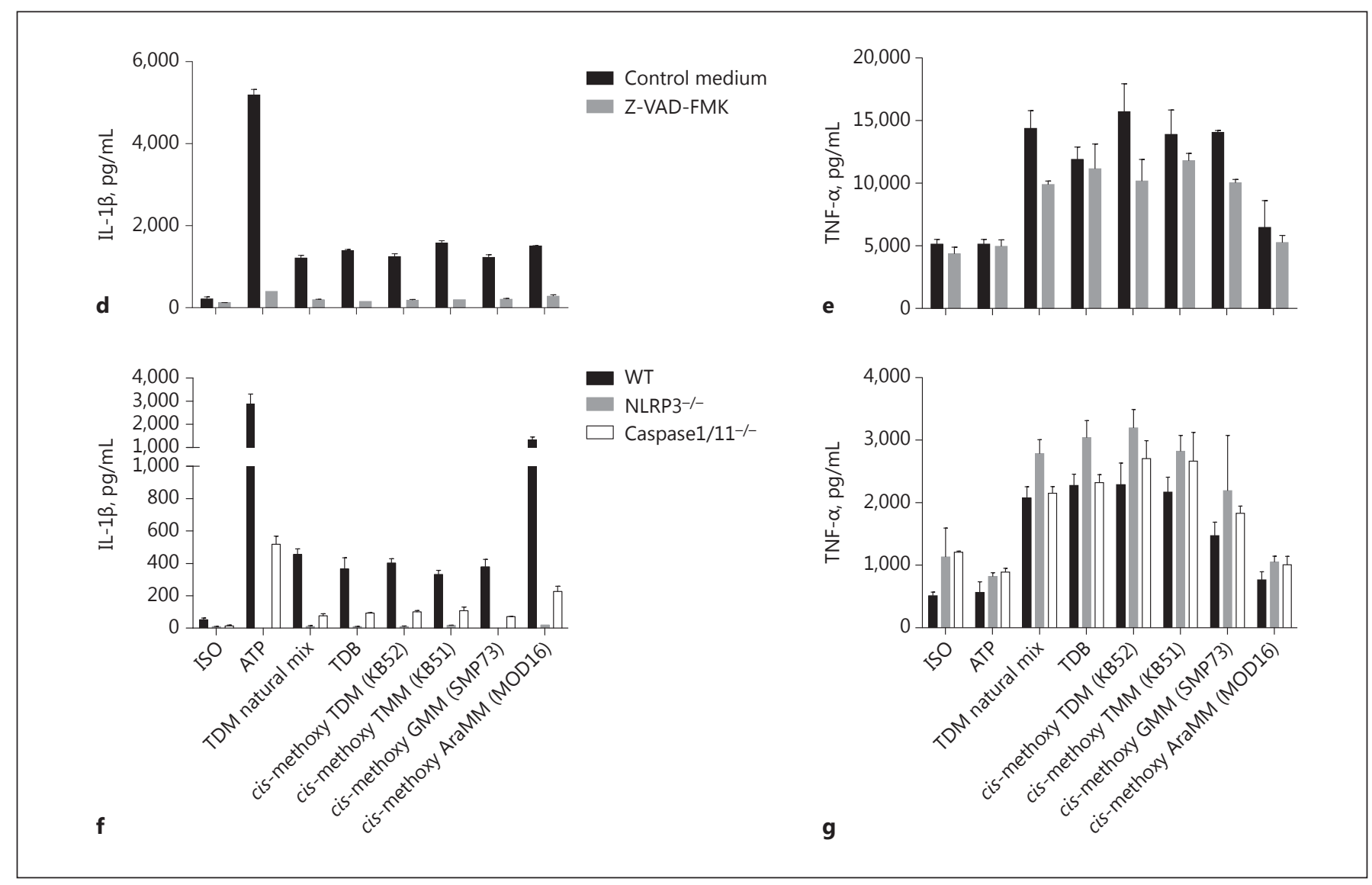

Fig. 4. Synthetic glucose and arabinose mycolate esters activate the NLRP3 inflammasome. a BMDCs prepared from C57BL/6 mice were primed for $3 \mathrm{~h}$ with $1 \mu \mathrm{g} / \mathrm{mL}$ of ultrapure LPS and then stimulated for $5 \mathrm{~h}$ with $10 \mu \mathrm{g} / \mathrm{mL}$ of synthetic mycolate esters, with evaporated ISO as a negative control and TDB or natural TDM mix at the same concentration as positive controls. The supernatants were harvested and the amount of IL- $1 \beta$ in the supernatants was determined by ELISA. $\mathbf{b}$ The secretion of active caspase- 1 p20 was detected by Western blot in the supernatant after methanol-chloroform precipitation. NS, non-specific band. c Quantification of caspase-1 p20 in the supernatant. The relative intensity of caspase-1 p20 was determined relative to a non-specific band used as a loading control in the supernatant. d, e BMDCs from C57BL/6 mice were primed with $1 \mu \mathrm{g} / \mathrm{mL}$ of ultrapure LPS for $2 \mathrm{~h}$, incubated with $10 \mu \mathrm{M}$ of Z-VAD-FMK or control medium for $1 \mathrm{~h}$ and stimulated with $10 \mu \mathrm{g} / \mathrm{mL}$ of synthetic mycolate esters and controls for $5 \mathrm{~h}$. Secretion of IL-1 $\beta$ and TNF- $\alpha$ was determined by ELISA in the supernatants. $\mathbf{f}, \mathbf{g}$ BMDCs were prepared from WT, $\mathrm{NLRP}^{-/-}$, and caspase-1/11 ${ }^{-/-} \mathrm{C} 57 \mathrm{BL} / 6$ mice and primed for $3 \mathrm{~h}$ with $1 \mu \mathrm{g} / \mathrm{mL}$ of ultrapure LPS before stimulation for $5 \mathrm{~h}$ with 10 $\mu \mathrm{g} / \mathrm{mL}$ of synthetic mycolate esters and controls. The amount of IL- $1 \beta$ and TNF- $\alpha$ in the supernatants was determined by ELISA. Results represent the mean \pm SD of triplicate cultures and are representative of at least 2 independent experiments. activation (Fig. 4g). As ROS production, potassium efflux, and cathepsin B activity are involved in inflammasome activation by TDB [23], we analysed whether similar mechanisms are also involved in BMDC activation by synthetic mycolate esters. To this end, production of IL- $1 \beta$ following incubation of BMDCs with ROS scavenger (Ebselen) and synthetic TDM-KB52, TMM-KB51, GMM-SMP73, and AraMM-MOD16 was measured. A significant reduction in IL- $1 \beta$ secretion was observed (see online suppl. Fig. S4A) following incubation with the dif- ferent compounds in the presence of Ebselen. The inhibition of IL-1 $\beta$ secretion was also observed when Glibenclamide was used as an inhibitor of potassium efflux pump or when a culture medium rich in potassium was used to inhibit potassium efflux (see online suppl. Fig. S4B). Finally, blocking cathepsin B activity with the pharmacological inhibitor CA-074 and blocking actin polymerisation with cytochalasin $\mathrm{D}$ significantly reduced the production of IL- $1 \beta$ by TDB and synthetic TDM-KB52, TMM-KB51, GMM-SMP73, and AraMM-MOD16 my- 
colate esters without affecting the level of IL- $1 \beta$ induced with the positive control ATP (see online suppl. Fig. S4C, D). In summary, these data indicate that ROS production, potassium efflux, cathepsin B activity, and phagocytosis are mechanisms involved in IL- $1 \beta$ induction by synthetic mycolate esters.

Synthetic Mycolate Esters Demonstrate Inflammatory and Adjuvant Properties in vivo

Our in vitro results indicated that synthetic TDMs and TMMs, GMMs, and to a lesser extent AraMMs had an inflammatory potential. To confirm these findings in vivo and to explore their possible use as novel adjuvants for subunit vaccines, w/o/w emulsions composed of IFA as a vehicle, synthetic mycolate esters, and ovalbumin as a test antigen were prepared and injected once in the 2 hind footpads of C57BL/6 mice. Footpad swelling was monitored for 1 week as a read-out of local inflammation; on day 7 we analysed the type of cells recruited in the footpad and the upregulation of proinflammatory chemokines and cytokines in these cells. Finally, OVA-specific immune responses were analysed by stimulating cells isolated from the lymph nodes draining the injection site 1 week after injection. Results obtained after injection of w/o/w emulsions composed of 30\% IFA and cis-methoxy TDM-KB52, TMM-KB51, GMM-SMP73, or AraMMMOD16 (10 $\mu \mathrm{g} /$ mouse) and ovalbumin (50 $\mu \mathrm{g} / \mathrm{mouse})$ are shown in Figures 5 and 6 . Responses were compared with those obtained with a vehicle control (30\% IFA + ovalbumin) or TDB w/o/w emulsions (30\% IFA, TDB 10 $\mu \mathrm{g} /$ mouse and ovalbumin $50 \mu \mathrm{g} /$ mouse). As shown in Figure 5a, neither AraMM-MOD16 nor 30\% IFA vehicle control induced any footpad swelling, while TDM-KB52, TMM-KB51, and GMM-SMP73 induced a footpad swelling of 1-2 mm over 7 days, comparable to that observed for a w/o/w emulsion with TDB. Interestingly, GMMSMP73 induced a significantly weaker footpad swelling than TDB. Cells recruited to the site of injection were analysed by flow cytometry. The analysis of cells recruited to the footpad showed a high infiltration of granulocytes (Ly6C+ and Ly6G+) for TDB, natural TDM, TDM-KB52, TMM-KB51, and GMM-SPM73, but not for AraMMMOD16 (Fig. 5b). qRT-PCR analysis confirmed that all the positive compounds (in terms of footpad swelling and granulocyte infiltration) also induced an increased expression of pro-inflammatory cytokines (IL-12p35, IL$1 \beta$, and TNF- $\alpha$ ) and chemokines (CXCL1) as compared to vehicle control and AraMM-MOD16 (Fig. 5c-f). No significant difference was observed between TDB, natural TDM, TDM-KB52, TMM-KB51, and GMM-SMP73. The

Inflammatory Properties and Adjuvant

Potential of Synthetic Mycolate Esters draining lymph node cell number increased 2-fold in animals injected with these 5 compounds, while the cell number was comparable in lymph nodes from animals injected with vehicle control or the AraMM-MOD16 group (Fig. 6a). Production of OVA-specific IL-2, IFN- $\gamma$, and IL-17A was measured in draining lymph node cell cultures stimulated in vitro with ovalbumin. Formulation with AraMM-MOD16 stimulated low levels of OVA-specific IL-2, IFN- $\gamma$, and no IL-17A production, whereas formulation with TDM-KB52, TMM-KB51, and GMMSMP73 induced elevated levels of these cytokines, comparable to those achieved by TDB (Fig. 6b-d).

Our analysis of the in vitro inflammatory properties of the synthetic mycolate esters had shown that the class of mycolate bound to a given sugar exerted only a small influence on the magnitude of the inflammatory response (Fig. 1). Nevertheless, there was a trend of the tested synthetic methoxy TDM to induce more TNF- $\alpha$ than TDB or the alpha- and keto-compounds. We therefore compared the in vivo responses of $\mathrm{w} / \mathrm{o} / \mathrm{w}$ emulsions composed of cis-alpha TDM-MH175 or TMM-MH176 with responses obtained with w/o/w emulsions composed of cis-methoxy TDM-KB52 or TMM-KB51. No differences between the cis-alpha and cis-methoxy mycolate esters could be observed in terms of footpad swelling and OVA-specific IFN- $\gamma$ and IL-17A responses (see online suppl. Fig. S5).

\section{Discussion}

The cell wall of mycobacteria is characterised by components that interact with different arms of the immune system in the case of infection. Several of these components have been associated with virulence and the capacity to modulate host immune responses. In this work, we were interested in analysing the role of esters of MAs using pure synthetic compounds. MAs are high molecular weight C-60 to C-90 2-alkyl-3-hydroxy fatty acids typical of mycobacteria. Different classes of MAs are present in mycobacteria, mainly alpha-, keto-, and methoxy-MAs, but also alkene and diene MAs. In addition, MAs can exist in different isomeric forms (e.g. cis- and trans-stereochemistry). In the mycobacterial cell wall, MAs are present as free MAs or esterified to different sugars (trehalose, glucose, or arabinose). TDM or cord factor is probably the most abundant and best studied of the non-cell wallbound mycolate esters, and several studies have reported that changing the class of MA in TDM influences its inflammatory properties. Indeed, TDM from the $M t b$ $\triangle c m a A 2$ mutant, lacking trans-cyclopropanation of 

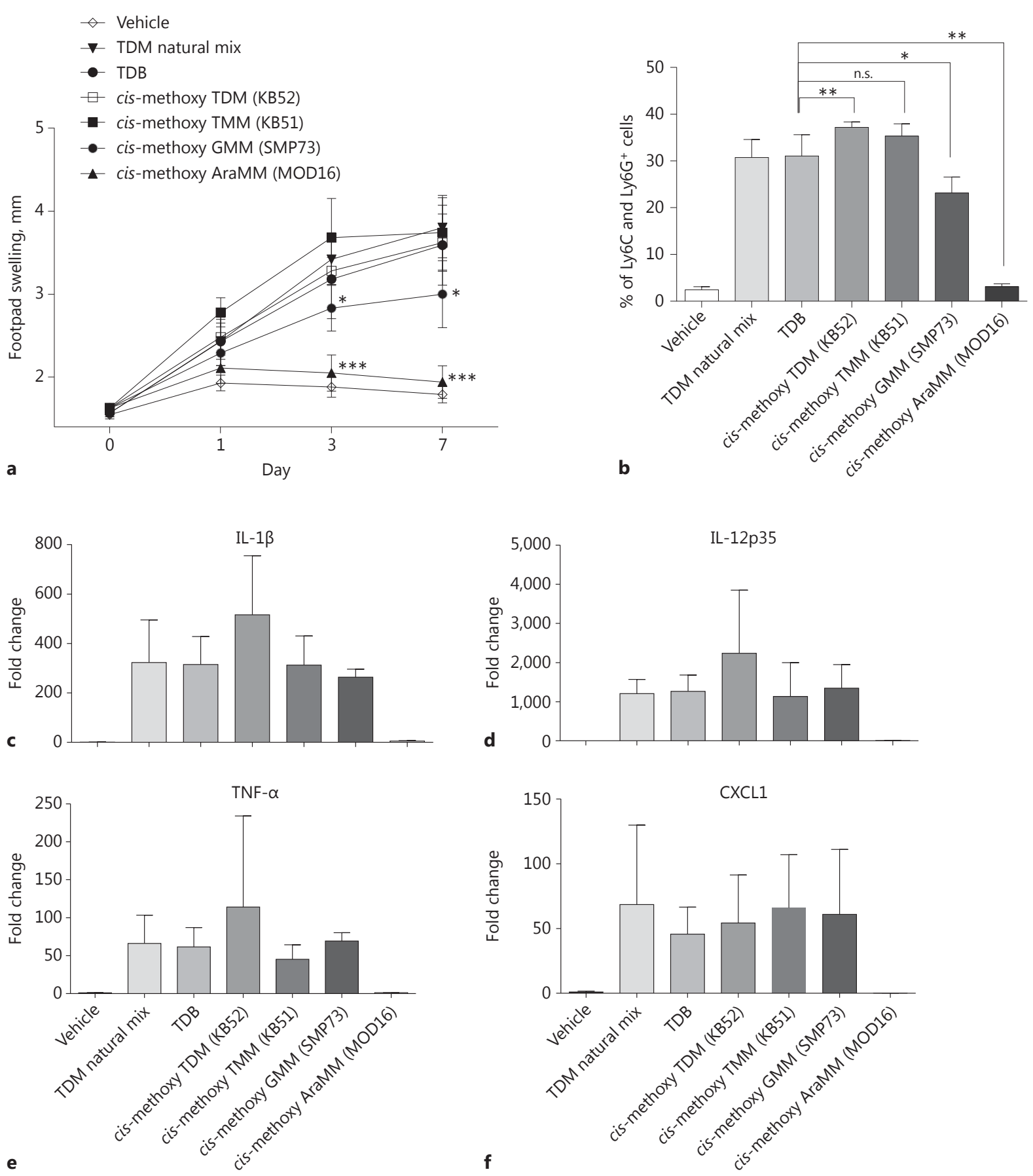

Fig. 5. In vivo inflammatory properties of synthetic mycolate esters. C57BL/6 mice were injected subcutaneously in the 2 hind footpads with w/o/w emulsions composed of $30 \%$ IFA, $10 \mu \mathrm{g} /$ mouse of specified synthetic mycolate esters, and $50 \mu \mathrm{g} / \mathrm{mouse}$ of ovalbumin. In the emulsion of the vehicle control group no glycolipid was present. In the TDB control group $10 \mu \mathrm{g} /$ mouse of TDB replaced the synthetic glycolipids. a Mean footpad size $(\mathrm{mm})$ of 5 mice tested individually was measured with a caliper on the day of injection and 1, 3, and 7 days after injection. $\mathbf{b}$ The percentage of

granulocytes (Ly6G+ and Ly6C+) was determined in cells isolated from the footpad by flow cytometry 7 days after administration. c-f The relative expressions of cytokines and chemokines were determined by qRT-PCR in the footpad cells. Fold changes were determined in comparison to vehicle. Footpad swelling and the percentage of granulocytes induced by synthetic mycolate esters were statistically analysed in comparison to TDB using the Mann-Whitney test. n.s., not significant. ${ }^{*} p<0.05,{ }^{* *} p<0.01$, and ${ }^{* * *} p<$ 0.001 . 


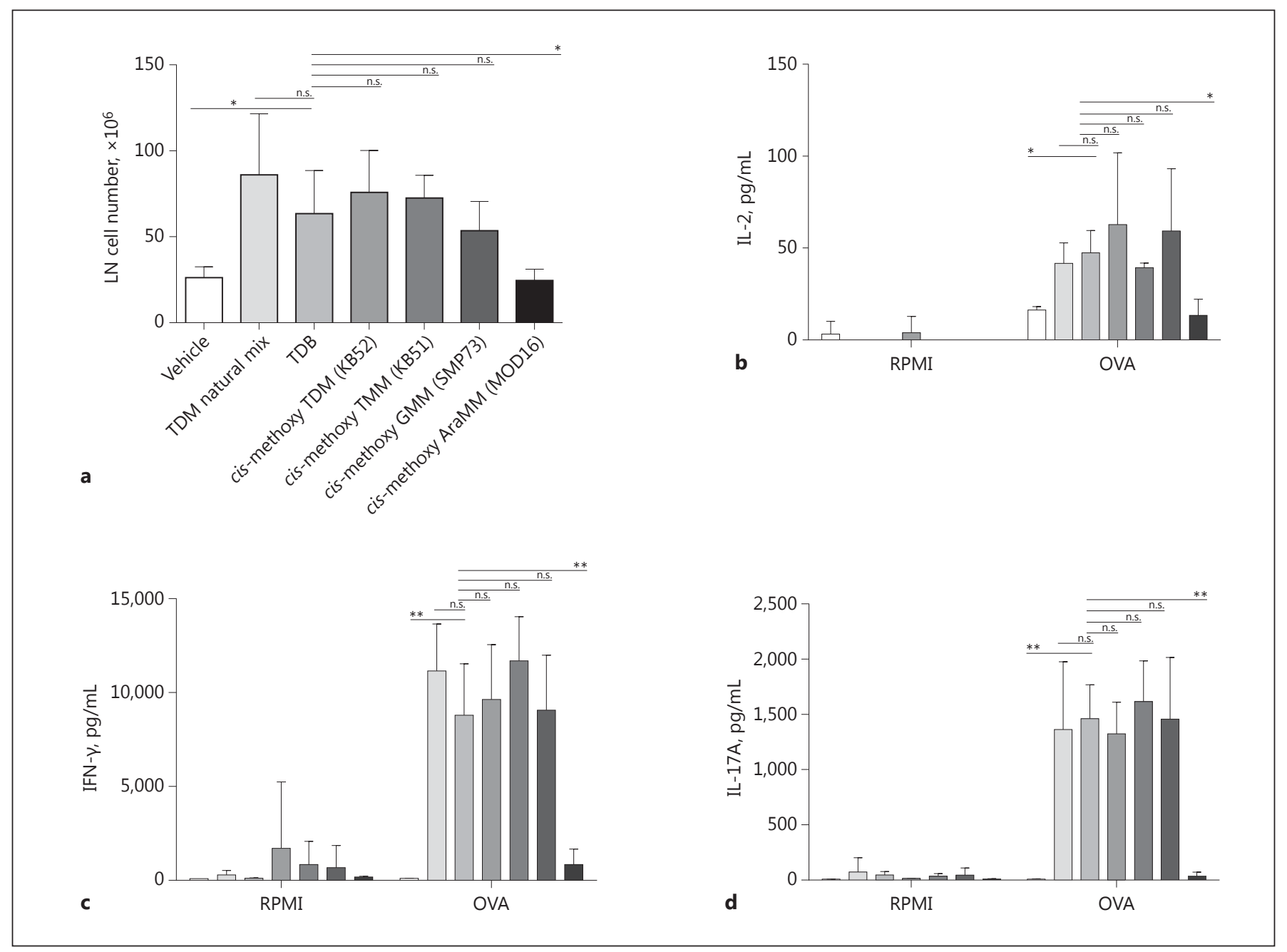

Fig. 6. In vivo adjuvant potential of synthetic mycolate esters in an experimental OVA model. a C57BL/6 mice were injected subcutaneously in the 2 hind footpads with w/o/w emulsions composed of $30 \%$ IFA, $10 \mu \mathrm{g} / \mathrm{mouse}$ of specified synthetic mycolate esters, and $50 \mu \mathrm{g} /$ mouse of ovalbumin. In the emulsion of the vehicle control group no glycolipid was present. In the TDB control group $10 \mu \mathrm{g} /$ mouse of TDB replaced the synthetic glycolipids. Mice were sacrificed 7 days after immunisation and cells from pooled popliteal

MAs, is hyperinflammatory for macrophages compared to TDM extracted from wild-type $M t b$ [36]. Likewise, the cord factor isolated from $M t b \Delta m m a A 4$, lacking oxygenated MA classes, induces in vitro more TNF- $\alpha$ and IL-12 in macrophages than TDM from wild-type $M t b$ [4]. On the other hand, TDM isolated from $M t b \triangle p c a A$ (an enzyme required for $\alpha$-mycolate cyclopropanation) is hypoinflammatory in macrophages [5]. In this study, we analysed the inflammatory power of 17 pure synthetic TDMs along with 13 TMMs, 5 GMMs, and 3 AraMMs. and inguinal lymph nodes were collected, counted (a) and stimulated with culture medium (RPMI) or $5 \mu \mathrm{g} / \mathrm{mL}$ of ovalbumin (OVA) for 24 or $72 \mathrm{~h}$. Supernatants were harvested and the concentrations of IL-2 (b), IFN- $\gamma(\mathbf{c})$, and IL-17A (d) were determined by ELISA. Results are means \pm SD of 5 mice tested individually and representative of at least 4 independent experiments. n.s., not significant. ${ }^{*} p<0.05$, and ${ }^{* *} p<0.01$ as determined by the MannWhitney test.
Our results only partially confirm the data previously obtained using these mutant $M t b$ strains $[4,5,36]$. Indeed, the monoalkene TDM and particularly the dialkene TDM, respectively lacking 1 and 2 cyclopropanes, show a lower inflammatory potential, confirming the findings of Rao et al. [5]. However, we found that the class of mycolate (alpha- vs. keto- vs. methoxy-) and the cis- versus trans-conformation only poorly influences the intensity of the inflammatory responses induced by these compounds. Cis-methoxy-mycolate esters seem to be more 
inflammatory compared to cis-alpha- and cis-keto-mycolate esters in vitro, but this observation was not confirmed in vivo. It is important to mention that the naturally isolated TDM preparations from $M t b$ mutants were composed of a complex mixture containing diverse mycolates in terms of class and chain length (C-60 to C-90), while the synthetic compounds tested in our study were of a particular class and generally restricted in chain length from C-74 to C-84. Synthetic compounds with chain lengths varying in a wider range between C-60 and C-90 need to be tested to make definitive conclusions. Moreover, Ostrop et al. [37] have shown that natural TDM induces a different profile of pro-inflammatory cytokines and chemokines compared to TDB in primary human APCs. These results suggest a potential role of chain length or presence of chemical groups in the activation of primary human cells. To elucidate this, it would be of great interest to compare the inflammatory potential of the synthetic compounds of our study in primary human APCs.

In addition, with this study we were able to demonstrate that besides TDMs and TMMs, GMMs and AraMMs are also inflammatory mycolate esters. Their ability to induce inflammatory responses is linked to their interaction with the C-type lectin receptor Mincle. Using BMDCs of $\mathrm{Mincle}^{-/-}$mice and human reporter cells expressing murine Mincle, we showed that long-chain mycobacterial GMMs - similarly to corynebacterial GMMs - can indeed interact with Mincle [19]. These results are different from those previously reported by Ishikawa et al. [18], who showed that GMM isolated by trehalase treatment of natural TDM does not interact with mouse Mincle receptor. This difference in results is possibly linked to the use of products of enzymatic treatment as compared to the use of pure synthetic compounds in our study and in the study by van der Peet et al. [19]. In addition, Matsunaga et al. [38], reported that, shortly after infection, mycobacteria have a downregulated production of TDM and an upregulated production of GMM. This reciprocal regulation is caused by competitive substrate selection by antigen $85 \mathrm{~A}$. The results reported by Matsunaga et al. [38], coupled to the results of Ishikawa et al. [18], led to the hypothesis that upregulation of GMM production is an escape mechanism of pathogenic mycobacteria to avoid recognition by the innate immune system. Our results suggest that even if in vivo a downregulation of TDM and an upregulation of GMM occur, innate immune cells remain able to recognise GMM via Mincle.

Regarding AraMMs, our results differ from what was described for natural AraMM purified from M. bovis
BCG, for which a dependence on TLR-2/Myd88 was reported [22]. However, in the report by Miyauchi [22], arabino-mycolate esters obtained by acid hydrolysis of BCG cell wall skeleton were used, which consist of a complex mixture of mono-arabinose monomycolates, tetra-arabinose tetra-mycolates, penta-arabinose tetra-mycolates and hexa-arabinose tetra-mycolate. The relative proportion of these different compounds in the preparation was not quantified; we can therefore not exclude that monoarabinose monomycolates signal via Mincle while the other arabinose mycolates would signal via TLR-2/ MyD88. It is noteworthy that, to our knowledge, this is the first study demonstrating that the C-type receptor Mincle can recognise pentose-based glycolipids, even if the intensity of the induced downstream signal was lower than the signal induced by trehalose- and glucose-based glycolipids.

In addition, our study demonstrates that, like TDMs, TMMs, and GMMs, AraMMs also induce the activation of the NLRP3 inflammasome. It should be noted that, even if the tested synthetic AraMMs were not as inflammatory as the synthetic TDMs, TMMs, and GMMs in the in vitro and in vivo experiments performed, in an in vitro model involving an LPS priming of BMDCs, a level of IL$1 \beta$ secretion was observed for all the tested AraMMs comparable to the levels observed for synthetic TDMs, TMMs, and GMMs. This indicates that synthetic AraMMs have a potential comparable to the other synthetic mycolate esters to induce NLRP3 inflammasome activation and, hence, pro-IL- $1 \beta$ processing. It is tempting to speculate that combining synthetic AraMM with a TLR-agonist could lead to the development of adjuvant formulations with Th1 and Th17 promoting potential. Indeed, the TLR agonist could trigger the production of pro-Th1 cytokines along with pro-IL-1 $\beta$ (similar to the LPS priming in our experimental set-up) and synthetic AraMM could lead to pro-IL- $1 \beta$ maturation by NLRP3 activation resulting in the induction of Th17 responses [24, 39].

The in vivo analysis of the tested synthetic mycolate esters in IFA formulations with ovalbumin confirmed the inflammatory properties of synthetic TDMs, TMMs, and GMMs as measured by footpad swelling following subcutaneous administration. No difference in terms of footpad swelling or OVA-specific T cell responses could be observed when comparing the cis-alpha TDM-MH175 or TMM-MH176 compounds to the cis-methoxy TDMKB52 or TMM-KB51 compounds, indicating that also in vivo the class of mycolate does not influence the inflammatory power of mycolate esters. Interestingly, Huber et al. [40] recently reported that trehalose monoesters with 
short acyl chains $(\mathrm{C} 12,14,16,18,20)$ induce significantly lower Th1 and Th17 responses compared to trehalose diester analogues. These results demonstrated that the adjuvant property of simple fatty acids requires 2 acyl chains. In contrast to that study, we observed comparable levels of Th1 and Th17 immune responses between TDMKB52 and TMM-KB51, or between TDM-MH175 and TMM-MH176, suggesting that, with a longer acyl chain, TMMs can also trigger strong immune responses in vivo. In contrast to what was reported by Shenderov et al. [39], purified peptidoglycan was not needed and formulation of these synthetic mycolate esters in IFA was sufficient in our hands to trigger antigen-specific Th17 responses along with Th1 responses. Differences in formulation and read-outs could account for this discrepancy. Our results indicate that synthetic TDMs, TMMs, and GMMs should be further evaluated as novel adjuvants for subunit vaccines to be used against infectious diseases for which Th1 and Th17 CD4+ T cell responses correlate with protection. However, it is known that natural TDM extracted from $M t b$ can be toxic in vivo [41]. Nevertheless, this toxicity is linked to the nature of the acyl chain bound to trehalose. Indeed, TDM from Rhodococcus sp. 4306 with a shorter chain length (C34-38) induces lower granulomatogenicity compared to TDM from $M t b$ [15]. In addition, using natural TDMs extracted from different mycobacteria species that vary in terms of the classes and proportions of mycolates present in their cell wall, Fujita et al. [42] showed that the classes of MA bound to trehalose can influence the toxicity of the molecule. Thus, toxicity of the synthetic compounds with an adjuvant potential described in this study should be assessed in vivo to downselect non-toxic compounds.

GMMs from mycobacteria are also protective nonprotein antigens recognised by CD1b-restricted $\mathrm{T}$ cells.
Hence, given this dual ability of GMMs to stimulate both innate and adaptive responses, it is tempting to speculate that administration of IFA formulations of synthetic GMMs with no additional adjuvant could stimulate protective immune responses. In future experiments it will be of interest to evaluate such formulations in animal models such as guinea pigs or human group 1 CD1 transgenic mice [43]. In addition, as already analysed for TMM [21] and in order to simplify the synthesis process, it would be interesting to determine the minimal carbon chain length necessary for the adjuvant potential of GMM and for its antigenic potential in tuberculosis vaccines. More importantly, this study raises the interesting possibility that GMM through Mincle and CD1b may contribute to both innate (induction of pro-inflammatory cytokines) and acquired (induction of Th1/Th17-CD1b-restricted $\mathrm{T}$ cell responses) immunity against mycobacteria. This fact provides an interesting possibility in the tuberculosis vaccine field to target both classical and non-classical $\mathrm{T}$ cell responses by combining synthetic GMM with a protective antigen.

\section{Acknowledgements}

Hermann Giresse Tima holds a FRIA PhD fellowship. This research was partially funded by the Interuniversity Attraction Pole Programme IAP 7/32 and by TBVAC2020 under grant agreement No. H2020-PHC-643381. MOM thanks the Ministry of Higher Education and Scientific Research, Kurdistan Region, Iraq, for the award of a PhD studentship. Roland Lang received support from the Deutsche Forschungsgemeinschaft (SFB 796/B6). We also thank Fabienne Jurion and Christophe van den Poel for technical support, and Virginie Maghe for advice concerning statistical analysis of the data.

\section{References}

1 Minnikin DE, Kremer L, Dover LG, Besra GS: The methyl-branched fortifications of $M y c o$ bacterium tuberculosis. Chem Biol 2002;9: 545-553.

2 Verschoor JA, Baird MS, Grooten J: Towards understanding the functional diversity of cell wall mycolic acids of Mycobacterium tuberculosis. Prog Lipid Res 2012;51:325-339.

3 Ojha AK, Baughn AD, Sambandan D, Hsu T, Trivelli X, Guerardel Y, et al: Growth of $M y$ cobacterium tuberculosis biofilms containing free mycolic acids and harbouring drug-tolerant bacteria. Mol Microbiol 2008;69:164-174.

Inflammatory Properties and Adjuvant Potential of Synthetic Mycolate Esters
4 Dao DN, Sweeney K, Hsu T, Gurcha SS, Nascimento IP, Roshevsky D, et al: Mycolic acid modification by the $m m a A 4$ gene of $M$. tuberculosis modulates IL-12 production. PLoS Pathog 2008;4:e1000081.

5 Rao V, Fujiwara N, Porcelli SA, Glickman MS: Mycobacterium tuberculosis controls host innate immune activation through cyclopropane modification of a glycolipid effector molecule. J Exp Med 2005;201:535-543.

6 Behling CA, Perez RL, Kidd MR, Staton GW, Hunter RL: Induction of pulmonary granulomas, macrophage procoagulant activity, and tumor necrosis factor-alpha by trehalose glycolipids. Ann Clin Lab Sci 1993;23:256-266.
7 Perez RL, Roman J, Staton GW, Hunter RL: Extravascular coagulation and fibrinolysis in murine lung inflammation induced by the mycobacterial cord factor trehalose-6,6-dimycolate. Am J Respir Crit Care Med 1994; 149:510-518.

8 Yarkoni E, Rapp HJ: Granuloma formation in lungs of mice after intravenous administration of emulsified trehalose- $6,6^{\prime}$-dimycolate (cord factor): reaction intensity depends on size distribution of the oil droplets. Infect Immun 1977;18:552-554. 
9 Axelrod S, Oschkinat H, Enders J, Schlegel B, Brinkmann V, Kaufmann SHE, et al: Delay of phagosome maturation by a mycobacterial lipid is reversed by nitric oxide. Cell Microbiol 2008;10:1530-1545.

10 Gavin AL, Hoebe K, Duong B, Ota T, Martin C, Beutler B, et al: Adjuvant-enhanced antibody responses in the absence of toll-like receptor signaling. Science 2006;314:19361938.

11 Watanabe M, Aoyagi Y, Ridell M, Minnikin DE: Separation and characterization of individual mycolic acids in representative mycobacteria. Microbiology 2001;147:1825-1837.

12 Minnikin DE, Parlett JH, Magnusson M, Ridell M, Lind A: Mycolic acid patterns of representatives of Mycobacterium bovis BCG. J Gen Microbiol 1984;130:2733-2736.

13 Dubnau E, Chan J, Raynaud C, Mohan VP, Lanéelle $\mathrm{M} \mathrm{a}, \mathrm{Yu} \mathrm{K}$, et al: Oxygenated mycolic acids are necessary for virulence of $M y c o b a c$ terium tuberculosis in mice. Mol Microbiol 2000;36:630-637.

14 Barkan D, Hedhli D, Yan H-G, Huygen K, Glickman MS: Mycobacterium tuberculosis lacking all mycolic acid cyclopropanation is viable but highly attenuated and hyperinflammatory in mice. Infect Immun 2012;80:19581968.

15 Ueda S, Fujiwara N, Naka T, Sakaguchi I, Ozeki Y, Yano I, et al: Structure-activity relationship of mycoloyl glycolipids derived from Rhodococcus sp. 4306. Microb Pathog 2001; 30:91-99.

16 Nishizawa M, Yamamoto H, Imagawa H, Barbier-Chassefière V, Petit E, Azuma I, et al: Efficient syntheses of a series of trehalose dimycolate (TDM)/trehalose dicorynomycolate (TDCM) analogues and their interleukin-6 level enhancement activity in mice sera. J Org Chem 2007;72:1627-1633.

17 Moody DB, Reinhold BB, Guy MR, Beckman EM, Frederique DE, Furlong ST, et al: Structural requirements for glycolipid antigen recognition by CD1b-restricted T cells. Science 1997;278:283-286.

18 Ishikawa E, Ishikawa T, Morita YS, Toyonaga K, Yamada H, Takeuchi O, et al: Direct recognition of the mycobacterial glycolipid, trehalose dimycolate, by C-type lectin Mincle. J Exp Med 2009;206:2879-2888.

19 van der Peet PL, Gunawan C, Torigoe S, Yamasaki S, Williams SJ: Corynomycolic acidcontaining glycolipids signal through the pattern recognition receptor Mincle. Chem Commun 2015;51:5100-5103.

20 Schoenen H, Bodendorfer B, Hitchens K, Manzanero S, Werninghaus K, Nimmerjahn F, et al: Cutting edge: Mincle is essential for recognition and adjuvanticity of the mycobacterial cord factor and its synthetic analog trehalose-dibehenate. J Immunol 2010;184: 2756-2760.

21 Stocker BL, Khan AA, Chee SH, Kamena F, Timmer MSM: On one leg: trehalose monoesters activate macrophages in a Mincle-dependant manner. Chembiochem 2014;15: 382-388.

22 Miyauchi M: Arabino-mycolates derived from cell-wall skeleton of Mycobacterium bovis $\mathrm{BCG}$ as a prominent structure for recognition by host immunity. Drug Discov Ther 2011;5:130-135.

23 Schweneker K, Gorka O, Schweneker M, Poeck H, Tschopp J, Peschel C, et al: The mycobacterial cord factor adjuvant analogue trehalose-6,6'-dibehenate (TDB) activates the Nlrp3 inflammasome. Immunobiology 2013; 218:664-673.

24 Desel C, Werninghaus K, Ritter M, Jozefowski K, Wenzel J, Russkamp N, et al: The Mincle-activating adjuvant TDB induces MyD88dependent Th1 and Th17 responses through IL-1R signaling. PLoS One 2013;8:e53531.

25 Mohammed M: Arabinose Mycolates from Synthetic Mycolic Acids; PhD thesis, Bangor University, Bangor, 2015.

26 Sahb MM, Dulayymi JR Al, Baird MS: Glucose monomycolates based on single synthetic mycolic acids. Chem Phys Lipids 2015;190: 9-14.

27 Al Dulayymi JR, Baird MS, Maza-Iglesias M, Hameed RT, Baols KS, Muzael M, et al: Synthetic trehalose di- and mono-esters of $\alpha$-, methoxy- and keto-mycolic acids. Tetrahedron 2014;70:9836-9852.

28 Al Dulayymi JR, Baird MS, Maza-Iglesias M, Vander Beken S, Grooten J: The first unique synthetic mycobacterial cord factors. Tetrahedron Lett 2009;50:3702-3705.

29 Ruland J, Duncan GS, Wakeham A, Mak TW: Differential requirement for Malt1 in T and B cell antigen receptor signaling. Immunity 2003;19:749-758.

30 Taher SG, Al Dulayymi JR, Baird MS: Synthetic trehalose esters of cis-alkene and diene $\alpha^{\prime}$-mycolic acids of Mycobacteria. Chem Phys Lipids 2015;189:28-38.

31 Baols KS: Synthesis of mixed cord factors and related compounds; $\mathrm{PhD}$ thesis, Bangor University, Bangor, 2014.

32 Inaba K, Inaba M, Romani N, Aya H, Deguchi M, Ikehara S, Muramatsu S, Steinman RM: Generation of large numbers of dendritic cells from mouse bone marrow cultures supplemented with granulocyte/macrophage colony-stimulating factor. J Exp Med 1992;176: 1693-1702.
33 Yarkoni E, Rapp HJ: Granuloma formation in lungs of mice after intravenous administration of emulsified trehalose-6,6'-dimycolate (cord factor): reaction intensity depends on size distribution of the oil droplets. Infect Immun 1977;18:552-554.

34 Miyake Y, Toyonaga K, Mori D, Kakuta S, Hoshino Y, Oyamada A, et al: C-type Lectin MCL is an FCR $\gamma$-coupled receptor that mediates the adjuvanticity of mycobacterial cord factor. Immunity 2013;38:1050-1062.

35 Schroder K, Tschopp J: The inflammasomes. Cell 2010;140:821-832.

36 Rao V, Gao F, Chen B, Jacobs WR, Glickman MS: Trans-cyclopropanation of mycolic acids on trehalose dimycolate suppresses Mycobacterium tuberculosis-induced inflammation and virulence. J Clin Invest 2006;116:16601667.

37 Ostrop J, Jozefowski K, Zimmermann S, Hofmann K, Strasser E, Lepenies B, et al: Contribution of MINCLE-SYK signaling to activation of primary human APCs by mycobacterial cord factor and the novel adjuvant TDB. J Immunol 2015;195:2417-2428.

38 Matsunaga I, Naka T, Talekar RS, McConnell MJ, Katoh K, Nakao H, et al: Mycolyltransferase-mediated glycolipid exchange in Mycobacteria. J Biol Chem 2008;283:2883528841.

39 Shenderov K, Barber DL, Mayer-Barber KD, Gurcha SS, Jankovic D, Feng CG, et al: Cord factor and peptidoglycan recapitulate the Th17-promoting adjuvant activity of mycobacteria through Mincle/CARD9 signaling and the inflammasome. J Immunol 2013;190: 5722-5730.

40 Huber A, Kallerup RS, Korsholm KS, Franzyk H, Lepenies B, Christensen D, et al: Trehalose diester glycolipids are superior to the monoesters in binding to Mincle, activation of macrophages in vitro and adjuvant activity in vivo. Innate Immun 2016;22:405-418.

41 Hunter RL, Armitige L, Jagannath C, Actor JK: TB Research at UT-Houston - a review of cord factor: new approaches to drugs, vaccines and the pathogenesis of tuberculosis. Tuberculosis 2009;89:1-20.

42 Fujita Y, Okamoto Y, Uenishi Y, Sunagawa M, Uchiyama T, Yano I: Molecular and supramolecular structure related differences in toxicity and granulomatogenic activity of mycobacterial cord factor in mice. Microb Pathog 2007;43:10-21.

43 Felio K, Nguyen H, Dascher CC, Choi H, Li S, Zimmer MI, et al: CD1-restricted adaptive immune responses to Mycobacteria in human group 1 CD1 transgenic mice. J Exp Med 2009;206:2497-2509. 